\title{
Early Brain Tumour Prediction using an Enhancement Feature Extraction Technique and Deep Neural Networks
}

\author{
S. Somasundaram, R. Gobinath
}

\begin{abstract}
Early tumor detection in the brain plays a vital role in early tumor diagnosis and radiotherapy planning. Magnetic resonance imaging (MRI) is latest technique which normally used for assessment of the brain tumor in Hospitals or scan centers. MRI images are used as the input image for brain tumor detection and classification. For predicting brain tumor earlier, an enhancement feature extraction technique and deep neural network are proposed. At first, the MRI image is pre-processed, segmented and feature extracted using image processing techniques. Support Vector Machine (SVM) based brain tumor classifications were performed previously with less accuracy rate. By using DNN classifier, there will be an improvement in accuracy rate. The proposed method mainly focuses on six features that are entropy, mean, correlation, contrast, energy and homogeneity. The performance metrics accuracy, sensitivity, and specificity are calculated to show that the proposed method is better compared to existing methods. The proposed technique is used to detect the location and the size of a tumor in the brain through MRI image by using MATLAB.

Keywords:Brain Tumor; Magnetic Resonance Imaging; Deep Neural Network; deep learning NN classifier; performance evaluation.
\end{abstract}

\section{INTRODUCTION}

The human brain is possibly the very important organ which monitors all our functional activities that are physical and mental. A brain tumor is defined as any unusual growth of cells in or around the brain. The main goal is to find a tumor location in the brain at an early stage. Doctor's take different types of images of the human brain like MRI, PET, etc. and analyze images to diagnose the tumor. It is very tough to differentiate between tumor and other substances appearing like a tumor inside the human brain. So, that the brain tumor identification becomes a challenging task.

Many diagnostic imaging methods likely executed for early detection of brain tumors, Positron Emission Tomography (PET), Computed Tomography (CT)and MRI. MRI technique is the most efficient method for detection and identification of brain tumor compared to the all other methods. One of the advantages of this technique is that it does not produce any dangerous radiation. This method has a

Revised Manuscript Received on July 08, 2019.

Mr. S. SOMASUNDARAM.R*, research Scholar Department of ComputerScience, VISTAS,Chennai, Tamil Nadu, India. srisaitechnologymadurai@gmail.com

Dr. R. GOBINATH.Assistant Professor, Department of Computer Science, VISTAS,Chennai, Tamil Nadu, India high contrast of soft tissues, and it is a non-invasive technique. Data is collected with functional structures, and brain tumor segmentation which gives potential irregular tissues on MRI. For best identification of a brain tumor, the Chan-vese clustering algorithm is proposed using segmentation and normalized histogram. Efficient classification of the MRIs was done by Deep Convolutional Neural Network (CNN) to provide accurate calculation and classification.

\section{LITERATURE VIEW}

Lei Mao et al. (2018) suggested a fully automatic segmentation method.The proposed technique inquired about a profound learning convolutional neural system which comprised of two techniques. One was Tumor Limitation Network (TLN) and the second one was Intra Tumor Classification Network (ITCN).. Later ITCN was used to mark the outlined tumor areas into many sub-regions. The proposed methodology gave efficient results than previous methods and increased the speed of segmentation. It provided better DSC values and complete segmentation task at the rate of 1.54 seconds per slice.

Zhu, J. (2018)proposed a new Conventional Neutral Network (CNN) model, Crossbar-Net, which consisted of two main innovations. One was the use of crossbar patches, and other was the design of cascaded boosting straining style with a cover re-sampling strategy. The crossbar patches covered kidney tumor in both vertical and horizontal directions and captured the local and contextual information concurrently. The suggested Crossbar-Net, which is the segment result of one sub model complemented by fine-tuning the other sub model, and each sub-model have performed self-improvement with re-sampling the mis-segmented region.

Larochelle et al. (2017) designed full automatic brain tumor segmentation using a deep neural network (DNN). The suggested systems were couturier to glioblastomas imagined in MR pictures. The basic idea was to find tumors having any shape, size, and differentiation anywhere in the brain. Here, the proposed technology gave a representation of various model decisions that were detected to be essential for acquiring focused execution. The survey was conducted specially for amazing designs because of Convolutional Neural Networks (CNN), i.e. DNNs DNNs predominantly adjusted to picture information. The current network of the conventional neural network is 
completely different from the previously used computer version. The current technology can provide 30 times faster than the previous technology.

\section{PROPOSED METHODOLGY}

Digital image processing technique is shown in fig 1 . According to the block diagram, MRI images as the input images. These images are given as input to the pre-processing block. In this stage, images are pre-processed which means that the size and shape of the images are intensified. The output of the pre-processing stage images is given as input to the image segmentation block. In this stage, segmentation is done using the Chan-Vese algorithm. The output images of image segmentation was given as input to the feature extraction block. In the feature extraction stage, the images are extracted for better accuracy, and it is done using Principle component analysis (PCA) technique and Gray Level Co-occurrence Matrix (GLCM) techniques. After feature extraction classification process is done, different classification techniques are used such as an artificial neural network, deep neural network, conventional neural network, etc. like. Here, classification is done using Deep Conventional Neural Network (CNN). Next step of after classification is the validation of result images. In this stage, resultant images are obtained. Proposed methodology is based on the DCNN learning architecture for classification and the classifier is used to identify brain tumors in brain MRI images.

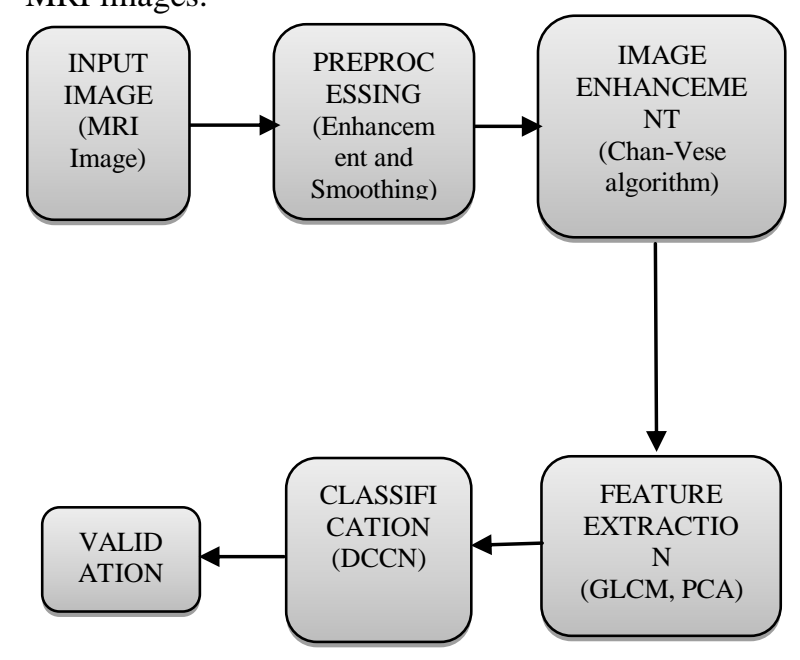

\subsection{PRE-PROCESSING}

\section{A. Enhancement}

Enhancement is improvement in quality and in medical imaging is used to make image clearer and to confirmbest presentation of all digital computer processing. It is more useful and significant to the medical diagnosis. Images can be interpreted by image processing. Enhancement can be achieved by removing noise, enhancing contrast, highlighting edges and modifying shapes.

\section{B. Smoothing}

Smoothing is done by noise removal, test removal and shaping of the image. If the image is in color then the image is resized and converted into grey scale. Bilateral filter is used to remove noise in the image. Bilateral filter is non-linear, edge protecting and noise reducing smoothing filter for all images. Bilateral Filter substitutes the intensity of each pixel with a weighted average of intensity values from nearby pixeland weightbased on Gaussian distribution.

The bilateral filter is defined as

$$
I^{\text {filtered }}(x)=\frac{1}{W_{p}} \sum_{x_{i} \in \Omega} I\left(x_{i}\right) f_{r}\left(\left\|I\left(x_{i}\right)-I(x)\right\|\right) g_{s}\left(\left\|x_{i}-x\right\|\right)
$$

where the normalization term

$$
W_{p}=\sum_{x_{i} \in \Omega} f_{r}\left(\left\|I\left(x_{i}\right)-I(x)\right\|\right) g_{s}\left(\left\|x_{i}-x\right\|\right)
$$

confirms that the filter preserves image energy and $I^{\text {filtered }}$ is the filtered image;

$I$ is the original input image to be filtered;

$x$ are the coordinates of the current pixel to be filtered;

$\Omega$ is the window centered in;

$f_{r}$ is the range kernel for smoothing differences in intensities (this function can be a Gaussian function)

$g_{s}$ is the spatial kernel for smoothing differences in coordinates (this function can be a Gaussian function).

The reshaped images are in the $256 \times 256$ range. The result of this phase is the distortion-free clear image having high contrast and quality.

\subsection{IMAGE SEGMENTATION}

Image segmentation is the process of dividing an image into sets of pixels which is also called superpixels. The main aim of segmentation is to identify the location of the tumor. In the proposed method, the Chan-Vese algorithm is used for segmentation.

Chan-Vese model for active outlines is a powerful and flexible method which can segment many types of images. It segmentimages which is difficult to segment in means of "classical" segmentation, using thresholding or gradient-based methods. The model is based on an energy minimization problem, which can be reformulated in the level set formulation, an easier way to solve the complex problems.

Let $\Omega$ be a bounded open set of $R^{2}$, with $\partial \Omega$ itsboundary.

Let $u_{0}: \Omega \rightarrow R$ be a given image, and $C(s)$ is a piecewise $C^{1}[0,1]$ parameterized a curve. Let's denote the region inside $C$ as $\omega$, and the region outside $C$ as $\Omega / \omega$ Additionally $c_{1}$ will denote the average pixels' intensity inside $C$, and $c_{2}$ will denote the average intensity outside $C\left(\right.$ i.e. $\left., c_{1}=c_{1}(C), c_{2}=c_{2}(C)\right)$.

$\inf _{c_{1}, c_{2}, C} F\left(c_{1}, c_{2}, C\right)$ 
The proposed algorithms provide best results as compared with the previous segmentation methods.

\section{FEATURE EXTRACTION}

Feature extraction targets to decrease the original data by calculating specific properties that differentiate one input pattern from another pattern. In this stage, the feature is extracted using the hybrid of PCA and GLCM.

$$
y=A\left(x-m_{x}\right)
$$

This point of view enables to form a simple formula (1), but it is necessary to keep in the mind that each row of the vector $x$ consists of $K$ values belonging to one input. The vector $m_{x}$ in Eq. (5) is the vector of mean values of all input variables defined by the relation

$$
\begin{aligned}
& m_{x}=E\{x\}=\frac{1}{k} \sum_{k=1}^{K} x_{k} \\
& N[i, j]=\frac{P[i, j]}{\sum_{i} \sum_{j} P[i, j]}
\end{aligned}
$$

Before this feature extraction, Dual-Tree Complex Discrete Wavelet Transform is used as segmentation pre-processing.

$$
x(t)=\sum_{n=-\infty}^{\infty} c(n) \phi(t-n)+\sum_{j=0}^{\infty} \sum_{n=-\infty}^{\infty} d(j, n) 2^{j / 2} \psi\left(2^{j} t-n\right)
$$

The scaling coefficients $c(n)$ and wavelet coefficients $d(j, n)$ are computed via the inner products.

$$
\begin{aligned}
& c(n)=\int_{-\infty}^{\infty} x(t) \phi(t-n) d t \\
& d(j, n)=2^{j / 2} \int_{-\infty}^{\infty} x(t) \psi\left(2^{j} t-n\right) d t
\end{aligned}
$$

They provide a time-frequency analysis of the signal by measuring its frequency content (controlled by the scale factor $j$ ) at different times (controlled by the time shift $n$ ).

\section{CLASSIFICATION}

Classification increases the accuracy, and better information from the individual class is achieved by using textures. There are three types of CNN's Pooling, Convolutional, and Fully-concentrated layers. At a convolution layer, the previous layer's feature maps are convolved with learnable kernels and put through the activation function to form the output feature map. Each output map may combine convolutions with multiple input maps. In general, we have that

$$
x_{j}^{l}=f\left(\sum_{i \in M_{j}} x_{i}^{l-1} * k_{i j}^{l}+b_{j}^{l}\right.
$$

where $M_{j}$ is a selection of input maps, and the convolution is of the "valid" border handling type when implemented in MATLAB.

\section{RESULTS AND DISCUSSION}

For performance evaluation of the present method, the brain tumor detection on early stage is done. The resulting images are compared to the original images, then the location of a tumor in the brain is found. Resultant images of image pre-processing, image segmentation, image extraction, and classification are mentioned below. The input image is resized into $256 * 256$. Then the resized image is converted into the grayscale image as shown in fig2. Then applying the bilateral filter noise is removed the noise as shown in fig3. The then filtered image is segmented using the Chan-Vese algorithm. In the segmentation process, the brain tumor is extracted from the filtered image as shown in fig4

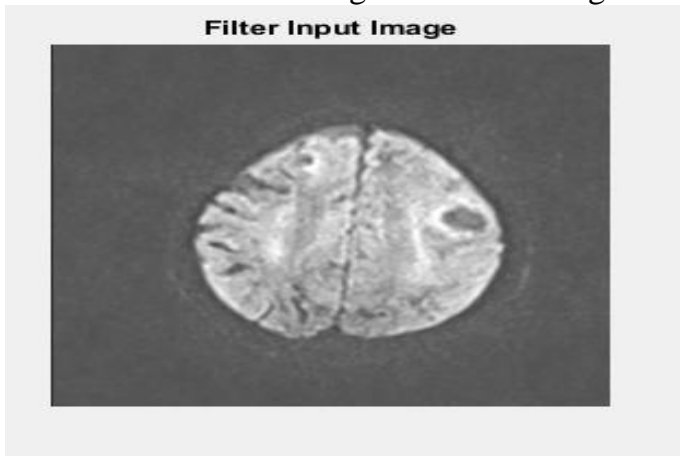

Figure 2 Resized image

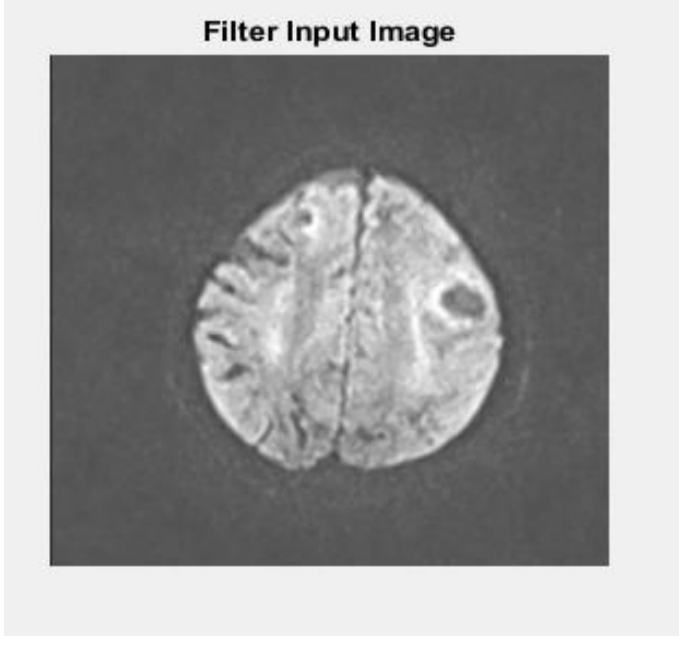

Figure 3 Filtered image

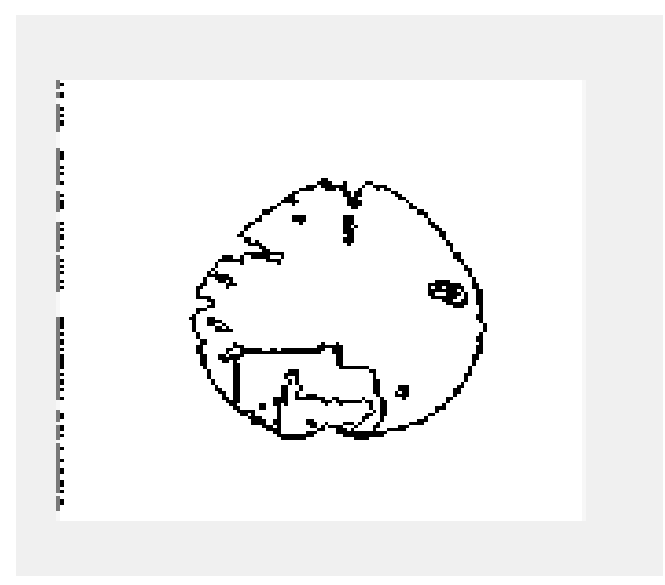

Figure 4 Segmented image

Then features are extracted from the segmented image using PCA

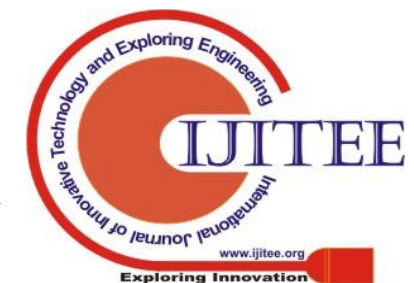


and GLCM. Extracted features are used as the input to the DCNN for the classification process. DCNN neural networks are shown in the below fig.

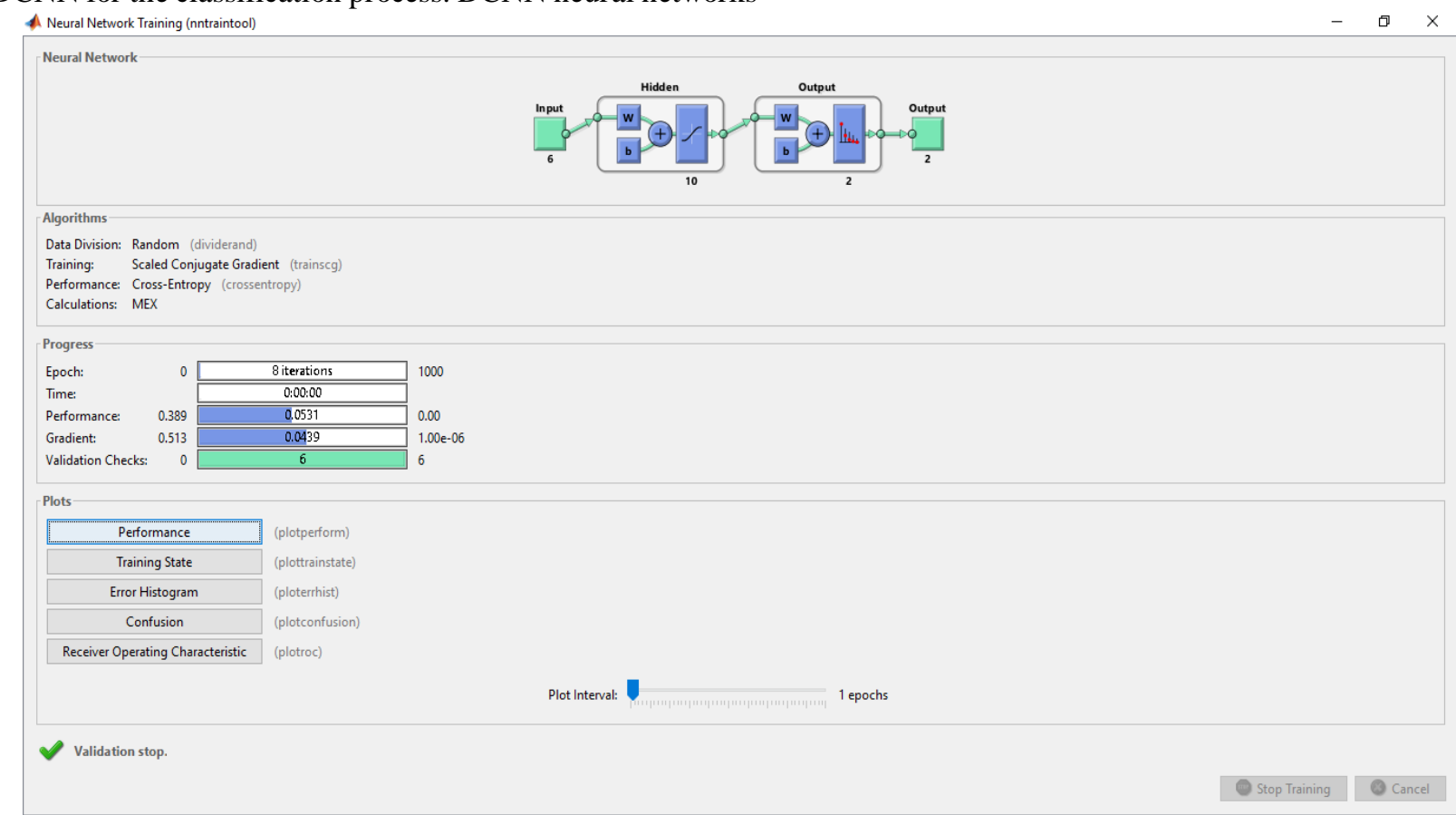

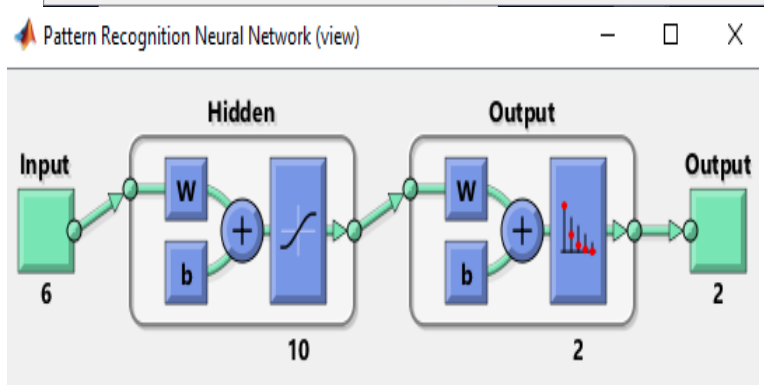

Figure 6 Neural networks

From these neural networks, the images are classified into four types. When we put the first-grade image as input, it will classify the image as shown in below dialogue box.

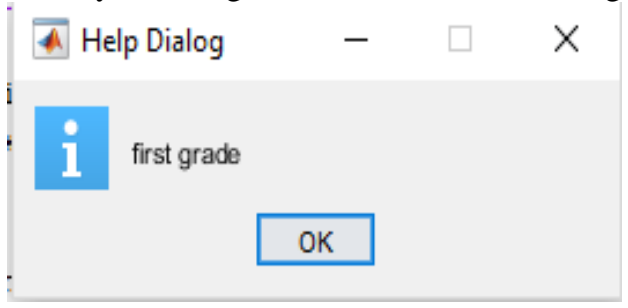

Figure 7 First-grade image

When we put the second-grade image as input, it will classify the image as shown in below dialogue box

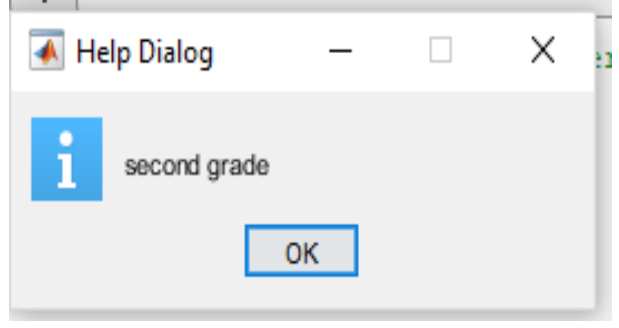

Figure 8 Second-grade image
When we put the third-grade image as input, it will classify the image as shown in below dialogue box.

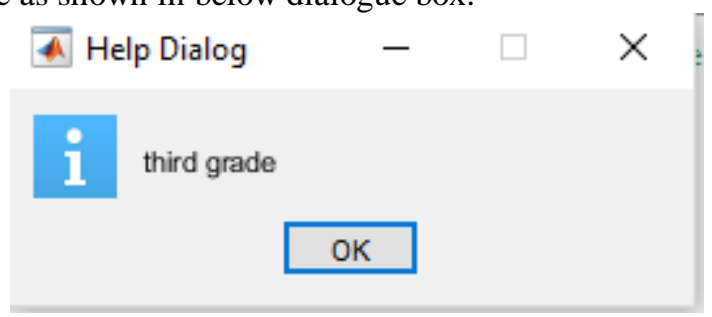

Figure 9 Third-grade image

When we put the fourth-grade image as input, it will classify the image as shown in the below dialogue box.

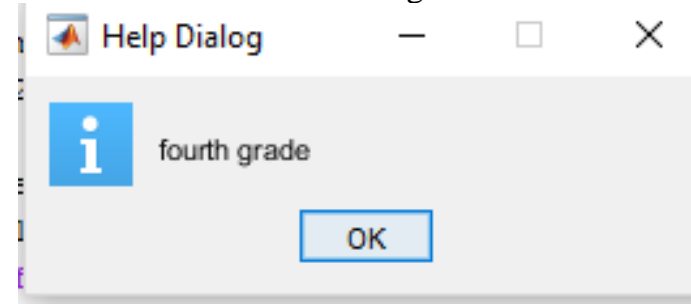

Figure 10 Fourth-grade image

\section{CONCLUSION}

The proposed methodology of early tumor detection in the brain plays a critical role in early tumor diagnosis and radiotherapy planning. In this study, MRI images were used as the input image for brain tumor detection and classification. An early brain tumor detection and classification was proposed based on the deep neural network. Initially, the MRI image was pre-processed, segmented and feature extracted using the techniques that were included in image processing and then classified using deep learning NN classifier. The performance metrics like accuracy, sensitivity, and specificity were calculated, and the calculations proved that the 
proposed method was better compared to existing methods. The accuracy rate is improved using DNN classifier. The same process is repeated for 50 images, and the results were found to be efficient.

\section{References}

1. Cui, S., Mao, L., Jiang, J., Liu, C., \&Xiong, S. (2018). Automatic Semantic Segmentation of Brain Gliomas from MRI Images Using a Deep Cascaded Neural Network. Journal of healthcare engineering, 2018.

2. Yu, Q., Shi, Y., Sun, J., Gao, Y., Dai, Y., \& Zhu, J. (2018). Crossbar-Net A Novel Convolutional Network for Kidney Tumor Segmentation in CT Images. arXiv preprint arXiv1804.10484.

3. Sharmila, R., \& Joseph, K. S. (2018). Brain Tumour Detection of MR Image Using Naïve Beyer classifier and Support Vector Machine.

4. Soltaninejad, M., Yang, G., Lambrou, T., Allinson, N., Jones, T. L., Barrick, T. R., ... \& Ye, X. (2018). Supervised Learning based Multimodal MRI Brain Tumour Segmentation using Texture Features from supervoxels. Computer Methods and Programs in Biomedicine.

5. Havaei, M., Davy, A., Warde-Farley, D., Biard, A., Courville, A., Bengio, Y., ... \& Larochelle, H. (2017). Brain tumor segmentation with deep neural networks. Medical image analysis, 35, 18-31.

6. Hemanth, D. J., Vijila, C. K. S., \& Anitha, J. (2010). Performance improved PSO based modified counter propagation neural network for abnormal MR brain image classification. Int. J. Advance. Soft Comput. Appl, 2(1), 65-84.

7. Kannan, S. R., Sathya, A., Ramathilagam, S., \& Devi, R. (2010). Novel segmentation algorithm in segmenting medical images. Journal of Systems and Software, 83(12), 2487-2495.

8. Lee, Y., Takahashi, N., Tsai, D. Y., \& Fujita, H. (2006, March) Detectability improvement of an early sign of acute stroke on brain CT images using an adaptive partial smoothing filter. In Proc. of SPIE Vol (Vol. 6144, pp. 61446Q-1).

9. Juang, L. H., \& Wu, M. N. (2010). MRI brain lesion image detection based on color-converted K-means clustering segmentation. Measurement, 43(7), 941-949.

10. Liao, C. C., Chiang, I. J., Xiao, F., \& WONG, J. M. (2006). Tracing the deformed midline on brain CT. Biomedical Engineering Applications, Basis, and Communications, 18(06), 305-311.

11. Liao, C. C., Xiao, F., Wong, J. M., \& Chiang, I. J. (2010). Computer-aided diagnosis of intracranial hematoma with brain deformation on computed tomography. Computerized medical imaging and graphics, 34(7), 563-571.

\section{AUTHORS PROFILE}

Mr. S. SOMASUNDARAM, Research Scholar Department of Computer Science, VISTAS,Chennai, Tamil Nadu, India

Dr. R. GOBINATH Assistant Professor, Department of Computer Science, VISTAS,

Chennai, Tamil Nadu, India 